\title{
Communications
}

\section{The Evolution of an Online Acquisitions System}

Jenko LUKAC: Lewis and Clark College Library, Portland, Oregon.

About two years ago a home-grown online acquisitions system was developed and implemented at Pacific University. The program, written in BASIC for the Data General Nova Computer, performs all the necessary functions such as ordering, receiving, fund accounting, etc. ${ }^{1}$ This program was offered to the library community, and about one hundred libraries from around the world have availed themselves of it.

One of the libraries that obtained and adopted Pacific's Electronic Acquisitions System (PEAS) was the Watzek Library at Lewis and Clark College. The advantage of a home-grown system is that it can be freely modified to suit the evolving needs of a particular library. This communication describes some of the changes made by Lewis and Clark College to the PEAS program, in order to illustrate how software developed at one institution can be "imported" into and enhanced by another institution. Although matters were particularly simplified by having the same person who developed PEAS at Pacific be responsible for the enhancements at Lewis and Clark, the procedure and conclusions are still generally applicable.

The first change made to the PEAS program was to rename it CLAS-the Computerized Library Acquisitions System. The most important change, however, was to translate it from Data General BASIC to Digital Equipment Corporation BASIC, since the computer at Lewis and Clark is a DEC VAX-11. (Each hardware manufacturer implements a slightly different version of a programming language.) The translation requires changing things such as square brackets to parentheses, the word read to get, the word write to put, etc. These changes would have to have been done repeatedly throughout the program, but, in fact, were quite easily accomplished by using a text editor-a metaprogram that can be instructed to change all occurrences of, for example, the word read to the word get in a single pass.

CLAS retained all of the features of PEAS, and became fully operational at Lewis and Clark in February of 1980. Since then, new features have been added as the staff expressed a need for them. Some are minor, such as having the computer recognize initial articles in titles. Others are more significant:

1. Searching for records in CLAS by author and title makes use of unlimited right- and left-handed truncation. This makes possible subject searching through key words in the title. For this purpose an extra terminal is provided at the reference desk.

2. CLAS permits the file to be searched by the name of the faculty member who requested the item, in addition to the eight other access points available in PEAS.

3. CLAS provides an activity report for any given period showing, for each fund, the amount ordered, the amount received, and the average cost per item.

4. CLAS can produce vendor reports showing for each vendor the average discount and the delivery schedule.

5. CLAS asks the operator to verify the cost of an item if the list price and cost differ by more than 30 percent.

6. CLAS allows the receipt of partial shipments.

Some of the enhancements to CLAS involved successive modifications. For example, one of the features of PEAS was the 
prevention of duplicate orders by matching new orders being input with records already in the database. A potential duplicate is reported if there is a match on both the author and the title fields. It was decided at the time of implementation at Lewis and Clark that this criterion was too restrictive, and CLAS was programmed to report a duplicate if only the title fields matched. After some months of experience, it turned out that even this requirement was excessively restrictive: a slight variation in the way a title was input would prevent a duplicate from showing up. The criterion was then further relaxed to signal duplicates if either the title or the author's last name matched. This, however, was too broad a net: although no duplicates were missed, ordering a book by Wilson or Smith produced a tedious list of potential duplicates. Hence, the requirement was tightened slightly to look for a match in either the title or the author's last name and first initial. This final criterion is currently serving well the needs of the Watzek Library. What is important about this evolutionary process is that it illustrates the dynamic way in which a library can "fine-tune" an automated system that is receptive to user modifications.

Since PEAS is supposed to be a selfexplanatory system, it lacks any documentation. CLAS is still a self-explanatory system, but nevertheless a manual has been produced to describe all its features and to record programming information such as the structure of the files. One version of the documentation is kept in machine-readable form so that it can be easily updated to correspond to developments in the program.

In conclusion, it can be stated that a library-application software package has been successfully transplanted from one institution to another, from one hardware environment to another, and in doing so has matured into a fuller and more flexible system, which it is hoped will, in turn, benefit other libraries contemplating the automation of their acquisitions operation. ${ }^{2}$

\section{REFERENCES}

1. Jenko Lukac, "A No Cost Online Acquisi- tions System for a Medium-Size Library," Library Journal 107:684-85 (March 15, 1980).

2. Interested libraries can request a copy of the CLAS program (\$80) or manual (\$40) directly from the author.

\section{The Significance of Information in the Ordinary Conduct of Life*}

Robert NEWHARD: Torrance Public Library, Torrance, California.

The information benefit provided to the general public by the developing telecommunications systems will be highly dependent upon the provider's perception of the current and potential role of information in the ordinary interests of life.

Assessing this role cannot easily be done by standard questionnaire or survey methods because information does not have a conscious function in people's lives.

Some paradigms from the past and present may, therefore, be of use in articulating the everyday importance of information.

\section{THE TOOL PARADIGM: INFORMATION AS A LINK BETWEEN MAN AND HIIS TOOLS OR REPAIRING A LOST CONFIDENCE}

Prior to the industrial revolution, most production was carried on in the home, using tools either made or repaired mainly at home. In this cottage industry, each person was very close to and secure in the use of his tools. With the advent of the industrial revolution and the factory system, the worker no longer owned his tools, but went to one place to use someone else's tools. Man and his tools began to separate. Many used the tools, fewer understood them. This process began to create the "expert."

Today most of the tools we use-the automobile, telephone, computer termi-

*A version of this paper was delivered at the meeting on "Public Libraries and the Remote Electronic Delivery of Information (REDI)," Columbus, Ohio, March 23-24, 1981. 